\title{
Integration of measurement and simulation of film pressure for estimating deformation of a glass sheet on a noncontact air conveyor
}

\author{
Rui YANG ${ }^{1,2}$, Wei ZHONG ${ }^{1,2 *}$, Rongyue WANG ${ }^{1,2}$, Chong $\mathrm{LI}^{1,2}$, Jiwen FANG ${ }^{3}$ \\ 1. School of Mechanical Engineering, Jiangsu University of Science and Technology, Zhenjiang, China \\ 2. Jiangsu Provincial Key Laboratory of Advanced Manufacturing for Marine Mechanical Equipment, Jiangsu University of Science and \\ Technology, Zhenjiang, China
}

* Corresponding author: Wei ZHONG, Email: zhongwei@just.edu.cn

\begin{abstract}
:
Recently, large and thin glass substrates are transported by air film conveyors to reduce surface damage. On the production line, the glass substrates are desired to be transported flatly on the conveyor to ensure the quality inspection. A method by feedbacking film pressure to the theoretical model is proposed for estimation of the deformation of the glass sheet, and the validity of the method is theoretically and experimentally verified. First, a theoretical model including the flow behavior through a porous-walled gap is established, and the film pressure distribution can be predicted by solving the model. Then, an experimental setup that can simultaneously measure the film pressure and the flatness of the glass sheet is established, and, the validity of the model is verified experimentally. Next, with the pressure points at the grooves as the boundary and the pressure points at the flange area as the feedback, an algorithm is applied to shape the one-dimensional deformation at the centerlines in accordance with a quadratic curve. Furthermore, two-dimensional deformation of the glass sheet can then be estimated by an interpolation operation. Comparisons of the calculated results with the experimental data verify the effectiveness of the estimating method.
\end{abstract}

Keywords: Deformation of glass sheet; air conveyor; air film; pressure distribution; pressure feedback

\section{Introduction}

Surface scratching and damage easily occur during transportation of glass substrates for liquid-crystal display (LCD). Contactless transport using pneumatic technology can eliminate these problems since air flow is magnetic free and clean ${ }^{[1-4]}$. For example, air tracks with arrays of bearing elements equipped on the surface are in use to supply pressurized air beneath flat objects. The air flow into the gap and form a pressurized film to levitate the object at a certain height, which is determined by the supply flow rate. This method is extremely suitable for the transport of large and fragile glass substrates.

Manufacturers intend to increase the size of the glass substrate to reduce $\operatorname{costs}^{[5]}$. The tenth generation thin flat panel glass substrate reaches a size of $3.1 \mathrm{~m}$ long by $2.8 \mathrm{~m}$ wide. On the production line, high-speed cameras are used to inspect the quality defects of the glass panel. Figure 1 gives a sketch of a noncontact transport system for inspecting the surface quality of the glass substrate. In the case that there exists an apparent deformation at some places, the detecting position cannot be clearly imaged due to the low focus depth of the camera. Figure 1 shows the situation that the deformation place cannot be clearly imaged. As a result, there is crucial need to monitor the deformation rapidly and accurately. Although multiple laser sensors can be used to achieve this, it is difficult to realize rapid measurement over a wide range.

Researchers have developed non-contact conveyor systems for transporting large glass substrates. Im et al. ${ }^{[6]}$ studied the influences from nozzles of different shapes to the air cushion and determined the optimum design. Amano et al. ${ }^{[7]}$ employed porous media to replace the orifice in order to eliminate the wave deformation. Oiwa et al. ${ }^{[8]}$ designed a contactless air conveyor using porous pads as restrictors, and reported the numerical results of the deformation of the glass sheet. Miyatake et al. ${ }^{\left[{ }^{[9]}\right.}$ studied the relation between the deformation and the supply pressure during transportation of the glass sheet. Above research indicate that the film pressure is closely related to the glass deformation. 
Finite element method (FEM) software can be used to calculate the deformation of the glass sheet. However, the calculation takes a long time, and, the actual practice needs a fast estimating method. Researchers sought methods to integrate the measured experimental data with the computation not only to enhance the calculation accuracy but also to reduce the time cost. ${ }^{[10-13]}$. Commonly, the calculation conditions are updated at time intervals based on past calculated results and measured data. Hayase $\mathrm{T}^{\text {[14] }}$ developed a simulation integrated experiment method to reproduce the real flow rate and analyzed the error.

Imagawa and Hayase ${ }^{[15]}$ introduced a measurementsimulation integration method for a turbulent flow to reduce the calculation consumption and also increase accuracy. Li et al ${ }^{[16]}$ indirectly estimated the suction force of a vacuum gripper (swirling air type) by measuring the film pressure distribution. Nakao et al ${ }^{[17]}$ suggested a measurement integrated simulation method to investigate unsteady flow through an orifice in a pipe.

This paper introduces an indirect method for predicting the deformation of the large, thin glass sheet by measuring film pressure. A theoretical model for calculating the film pressure is built, and the deformation curve is shaped to make the calculated result close to the measured pressure data. The method is adopted to estimate a two-dimensional deformation, and the effectiveness of the method is verified experimentally.

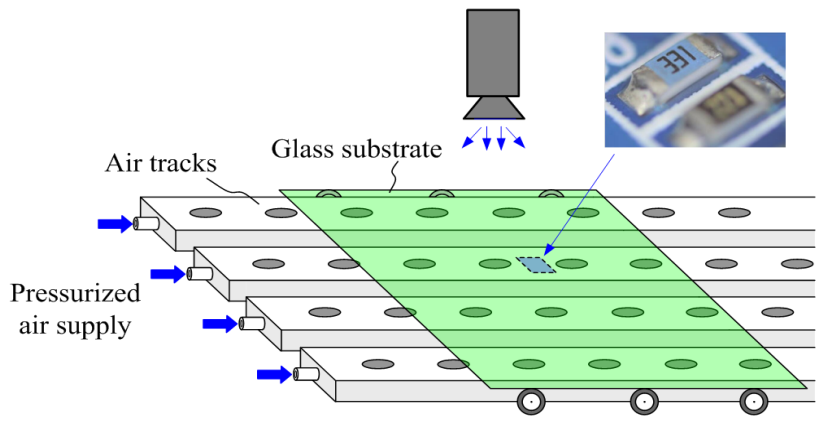

Figure 1 A noncontact transportation system of large glass panels for the inspection process

\section{Construction of the air conveyor}

Figure 2 shows a sketch of a porous pads equipped air conveyor system that has three conveyor lines. Multiple air conveyor lines are used to ease fabrication and adjustment. For those extremely large, thin glass sheets, air tracks with multiply array of porous pad can be used to produce a uniform film pressure distribution. Pressurized air is provided by the porous pads so that an air film can be formed under the glass sheet. With surface areas like sails, supporting force are formed to levitate the glass sheet on the conveyor. Such non-contact mode during the conveying process can keep the surface clean and prevent surface scratch which are easily caused by traditional method with mechanical contact. In this way, the glass panels are moved by the friction force provided by the installed rollers. In this application, the contact points for the rollers with the surface locate near the edge, and this would not damage the surface quality.

The geometry of a representative area of the noncontact air conveyor is also illustrated in figure 2 . Air supply units are regularly arranged on the conveyor surface. The unit is a square, with a length of $45 \mathrm{~mm}$. For each unit, a circular porous pad, which has a diameter of $20 \mathrm{~mm}$ and a length of $3 \mathrm{~mm}$, is placed at the center as the restrictor. Grooves ( $5 \mathrm{~mm}$ in width, $1 \mathrm{~mm}$ in depth) are set between the units to further uniform the film pressure and to case the stress concentration on the glass sheet. This treatment reduces the bearing capacity and stiffness of the air film; however, without the grooves, the air film would exhibit a large pressure concentrated at the center area, where the flow field would become too complicated to be analyzed.

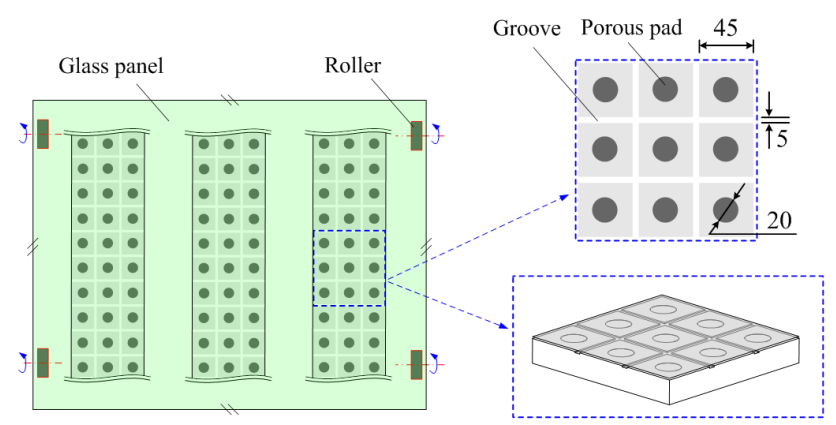

Figure 2 A sketch of an air conveyor system

\section{Film pressure distribution}

\subsection{Mathematical Modeling}

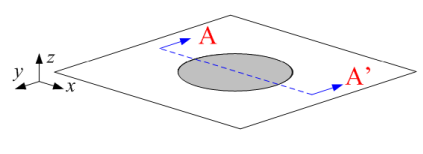

(a) A representative unit

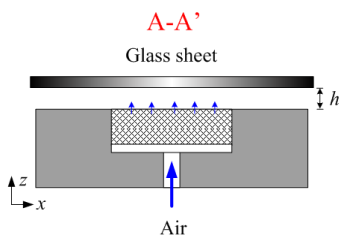

(b) flow in the gap
Figure 3 Airflow model

Figure 3 shows a representative unit, which is a square area including a porous pad as well as its surrounding flanges. A Cartesian coordinate is established, and the bottom left point of the gap of the unit is considered as the origin. To reduce the complexity of modeling of flow in the gap, we assume that the airflow is laminar (The Reynolds number is far below $10^{3}$ ) and film pressure change in $\mathrm{z}$ direction is ignored. With the assumption, a simplified Navier-Stokes equation in $x$-direction can be written as below.

$$
-\frac{\partial p}{\partial x}+\mu \frac{\partial^{2} u_{x}}{\partial z^{2}}=0
$$

where $p$ denotes the film pressure, $\mathrm{u}_{\mathrm{x}}$ denotes the flow velocity, and $\mu$ denotes the air viscosity.

In $\mathrm{z}$-direction, Navier-Stokes equation can be written as below 
$\mu \frac{\partial^{2} u_{z}}{\partial z^{2}}=0$

Assuming a no-slip case, the boundary condition that $\mathrm{u}_{\mathrm{x}}=0(\mathrm{z}=0)$ and $\mathrm{u}_{\mathrm{x}}=0(\mathrm{z}=\mathrm{h})$ can be obtained, and equation (3) is obtained by integrating equation (1) with respect to $z$ $u_{x}=\frac{1}{2 \mu} \frac{\partial p}{\partial x}\left(z^{2}-h z\right)$

where $h$ denotes the thickness of the gap.

Similarly, with the boundary velocity that $\mathrm{u}_{\mathrm{z}}=0(\mathrm{z}=0)$ and $\mathrm{u}_{\mathrm{z}}=0(\mathrm{z}=\mathrm{h})$, the flow velocity $u_{\mathrm{z}}$ can be determined by integrating equation (2) with respect to $z$,

$u_{z}=\omega_{0}-\frac{\omega_{0}}{h} z$

where $\omega_{0}$ is the porous walled outflow velocity. The velocity can be obtained by the flow regime through porous media ${ }^{[18,19]}$.

The continuity equation is expressed as below

$\frac{\partial\left(p u_{x}\right)}{\partial x}+\frac{\partial\left(p u_{z}\right)}{\partial z}+\frac{\partial p}{\partial t}=0$

Substituting equations (3) and (4) into equation (5), yields

$\frac{\partial p}{\partial t}=\frac{h^{2}}{12 \mu}\left(\frac{\partial p}{\partial x} \frac{\partial p}{\partial x}+p \frac{\partial^{2} p}{\partial x^{2}}\right)+\frac{p \omega_{0}}{h}$

The gap that filled by air should be divided into a number of grids so that the iteration calculation can be applied to obtain the film pressure. Figure 4 shows the generated grids of the calculation domain. The crosssectional area of the gap is divided into $\mathrm{n}$ grids, marked as $A[i](i=0-n)$. Figure 5 shows the procedure to calculate the film pressure. Initially, atmospheric pressure is assigned to each grid. Then, the outlet flow rate from the porous surface is calculated by the Forchheimer equation, and thus, the velocity $\omega_{0}$ is known. A finite volume method (FVM) is applied to solve equation (6) to obtain the pressure distribution. The Euler explicit method is used for iteration calculation, and the time step is $10^{-8} \mathrm{~s}$. The calculated data are updated and saved after every iteration cycle until the calculation converges. The final results can be obtained after the calculation converges. When the difference between the inlet flow rate and the outlet flow rate is less than $\pm 1 \%$, it can be considered that the calculation converges.

\subsection{Experimental apparatus}

Figure 6 shows a sketch of the apparatus used to measure the deformation of the glass sheet. An air conveyor with a size of $3 \times 3$ units is fabricated. A glass sheet with a size of $200 \mathrm{~mm} \times 155 \mathrm{~mm} \times 0.7 \mathrm{~mm}$ is supported on the conveyor. The glass sheet is with the following physical properties: 1 ) the density is $2500\left(\mathrm{~kg} / \mathrm{m}^{3}\right)$, 2) Young's modulus is $73 \mathrm{GPa}$, 3 ) the Poisson's ratio is 0.25 . The glass sheet is supported at four corners, and the vertical distance from the conveyor surface to the supporting point is $150 \mu \mathrm{m}$. A high resolution laser sensor is used to measure the deformation of the glass sheet. The laser sensor is fixed on an XY sliding table, the position of which is detected using two installed displacement sensors. The deformation can be measured by the following steps: (1) Place several plug gauges between the conveyor and the glass sheet, and measure the flatness without air supply. (2) Repeat the measurement with air supply. The difference between the two measurements approximates the deformation. Figure 6 shows the moving route of the laser sensor. The slide table moves at a velocity of $5 \mathrm{~mm} / \mathrm{min}$ for detecting the flatness along $\mathrm{x}$-direction, and, it moves in $5 \mathrm{~mm}$ increments without recording the data along $y$-direction.

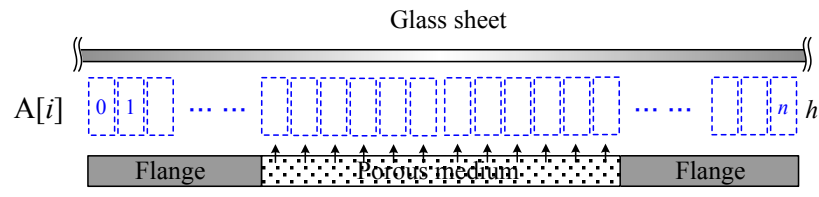

Figure 4 Grid generation of the computational domain

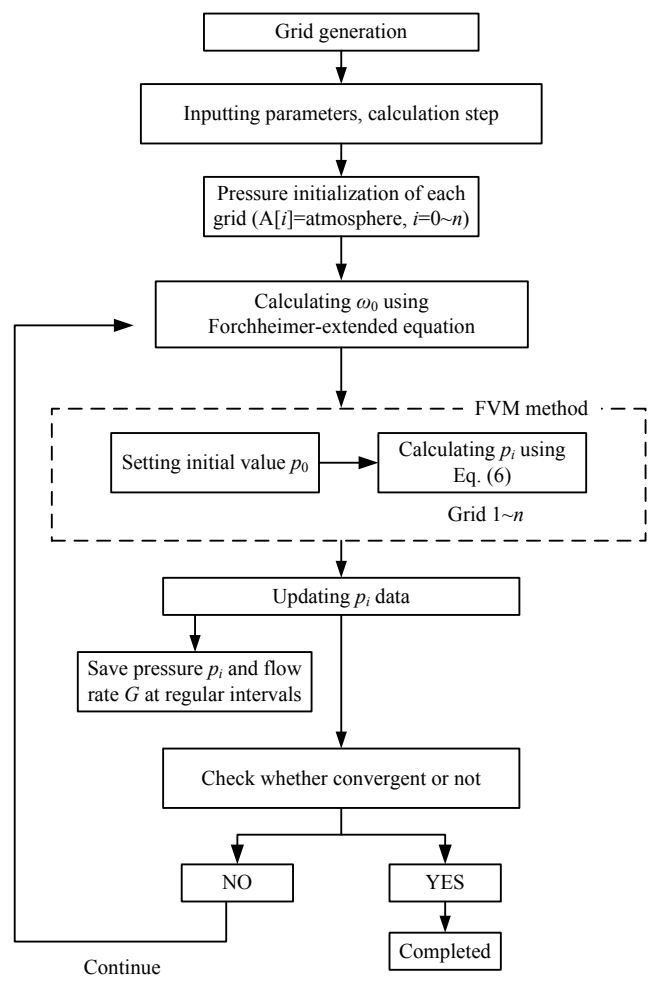

Figure 5 A flowchart indicating the calculation of air film pressure

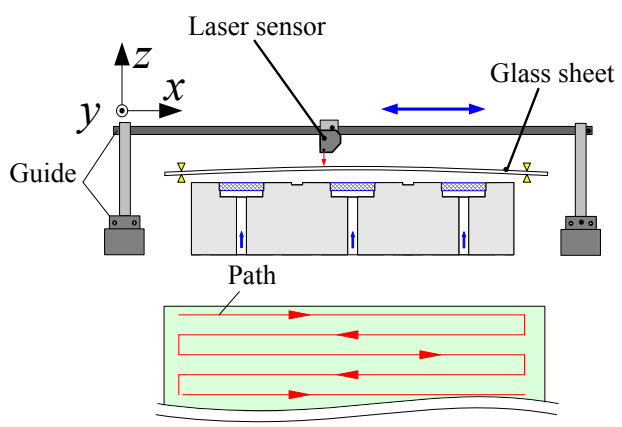

Figure 6 A sketch of the apparatus that shows the measuring method 


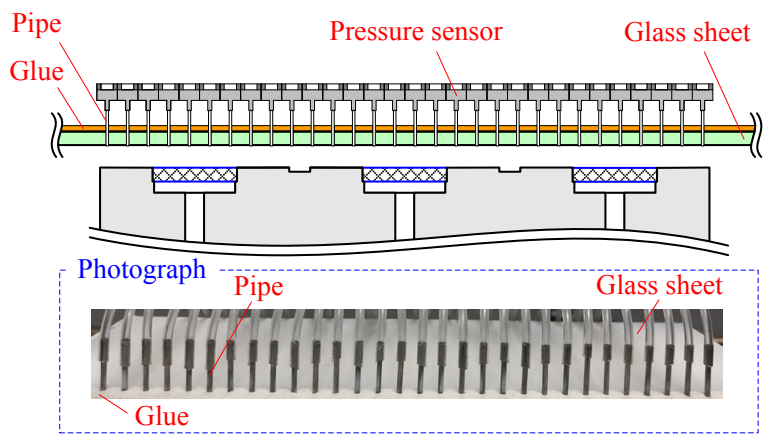
air film

Figure 7 Apparatus for measuring the pressure of the

The film pressure distribution is measured to verify the theoretical model. Previous researches ${ }^{[20,21]}$ reported several methods concerning measurement of the film pressure in the case that the object will not deform and the two surfaces are in parallel. However, in current work, the glass sheet appears an obvious deformation that cannot be ignored since it is very thin. To realize the measurement of the film pressure in a deformed status, the apparatus shown in figure 7 is used. Thirty through-holes are opened on the glass sheet. These holes are with a diameter of $2 \mathrm{~mm}$ and arranged at $5 \mathrm{~mm}$ intervals. Each hole is individually connected to a high resolution pressure sensor using a metallic tube. A glue layer is coated on the surface to seal the tubes. When we supply pressurized air via the porous pad, under the effect of the air film, the glass sheet is in a deformed status. The deformation of the glass sheet on the centerline was measured and investigated.

\subsection{Film pressure distribution}

Experiments are first performed to produce an extent of deformation and the film pressure is measured to verify the theoretical model. Here, the deformation and the film pressure on the centerline (AA' in figure 2) are discussed. The deformation versus the position is measured in two cases. The two cases are with different degrees of deformation (Figure 8). Case 1 shows that the glass sheet appears a certain degree of deformation and case 2 shows that the glass sheet is in parallel with the conveyor surface. The case 2 can be achieved by using a sufficiently thick glass sheet. The measured points are passed through by a quadratic fitting curve, which is treated as the real deformation. Thus, the film pressure can be calculated with the theoretical model stated above.

Figure 9 shows the measured film pressure in comparison with the calculated results for the two cases. The supporting points are at a $150 \mu \mathrm{m}$ height from the surface. The supply flow rate for each unit is set to $3 \mathrm{~L} / \mathrm{min}$. The film pressure at the grooves is detected and treated as the boundary to calculate the pressure distribution in each unit. The film pressure is distributed like a parabola, and the grooves uniform the film pressure of different units. Without these grooves, flow interference would appear in the gap, and this makes the flow pattern too complicated to be modeled. Existence of the pressure-equalizing grooves enables independent calculations of the film pressure for each unit, which greatly improves the computation efficiency. Observations on the results show that, for the case 1, the film pressure of the inner unit is slightly larger than that of the outer units, but for the case 2 where the center portion exhibits a larger deformation, the center pressure show a lower value compared with that of the outer area. Although the theoretical model is deduced with the assumption that the glass sheet is in parallel with the conveyor surface, the comparison between the calculated results and the experimental data indicates that the calculation can approximate the experimental film pressure when the deformation is limited within $0.1 \mathrm{~mm}$. It should also be noted that for the maximum deformation that is larger than $0.1 \mathrm{~mm}$ but smaller than $0.3 \mathrm{~mm}$, the modeling for the flat center portion might also be valid but not for the outer units.

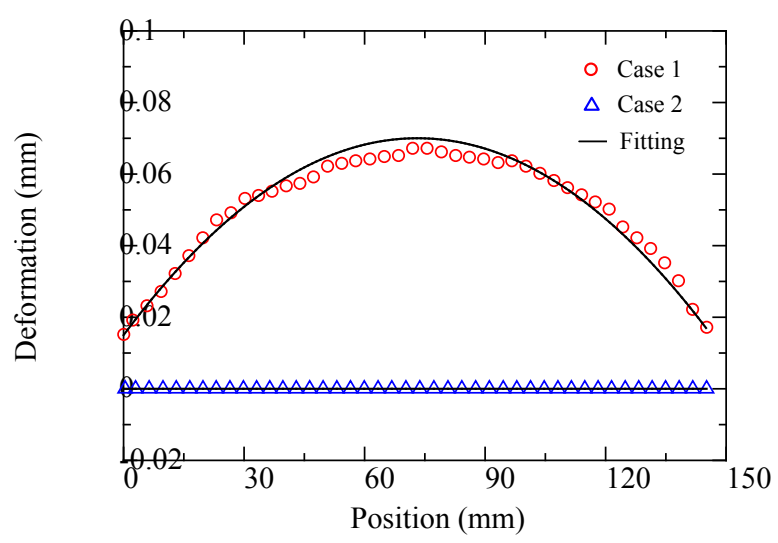

Figure 8 Fittings of the deformation results of two cases

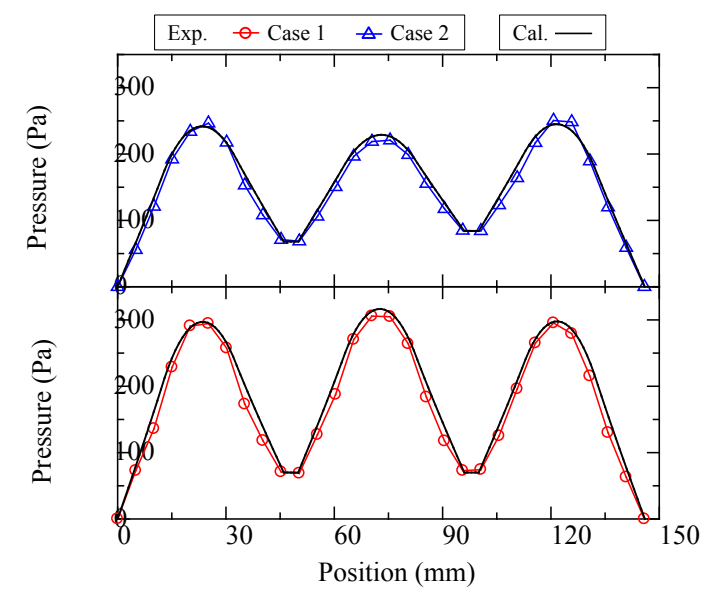

Figure 9 Film pressure distribution of the two cases

\section{Estimation of the deformation of the glass sheet and the results}

Figure 10 shows a one dimensional case where a glass sheet is supported at two points and deformed by the film pressure is considered. The vertical position of the 
supporting points is treated as the base. The distance of the conveyor surface relative to the base is denoted as $\mathrm{h}_{0}$. The maximum deformation (at the center) relative to the base is denoted as $h_{c}$. A quadratic curve expressed by $f(x)=a x^{2}+b x+c$ is used to shape the deformation with respect to the position $\mathrm{x}$. Here, $\mathrm{a}, \mathrm{b}$, and $\mathrm{c}$ are coefficients that need to be determined. The deflections at two sides are treated as the boundary conditions, and two position sensors are installed to detect the edge deflections, which are respectively denoted as $\delta_{1}$ and $\delta_{2}$. The left supporting point is regarded as the original point. Using three points on the curve, $\left(\mathrm{L}_{1}, \delta_{1}\right),\left(\mathrm{L} / 2, \mathrm{~h}_{\mathrm{c}}\right)$, and $\left(\mathrm{L}_{1}+\mathrm{L}_{2}, \delta_{2}\right)$, determines the coefficients $\mathrm{a}, \mathrm{b}$, and $\mathrm{c}$ for the deformation curve by the following equations

$$
\left\{\begin{array}{l}
a L_{1}^{2}+b L_{1}+c=\delta_{1} \\
a \frac{L^{2}}{4}+\frac{b}{2} L+c=h_{c} \\
a L_{1}^{2}+2 a L_{1} L_{2}+a L_{2}^{2}+b L_{1}+b L_{2}+c=\delta_{2}
\end{array}\right.
$$

By this method, the deformation along the centerline in the form of $f(x)=a x^{2}+b x+c$ can be obtained. The expression of $\mathrm{f}(\mathrm{x})$ with $\mathrm{L}_{1}=0.028 \mathrm{~m}, \mathrm{~L}_{2}=0.145 \mathrm{~m}$ and $\mathrm{L}=$ $0.2 \mathrm{~m}$ can then be determined as

$f(x)=\left(95.785 \delta_{1}-190.258 h_{c}+94.473 \delta_{2}\right) x^{2}+\left(-26.149 \delta_{1}+38.242 h_{c}-12.092 \delta_{2}\right) x$

$+\left(1.657 \delta_{1}-0.921 h_{c}+0.265 \delta_{2}\right)$

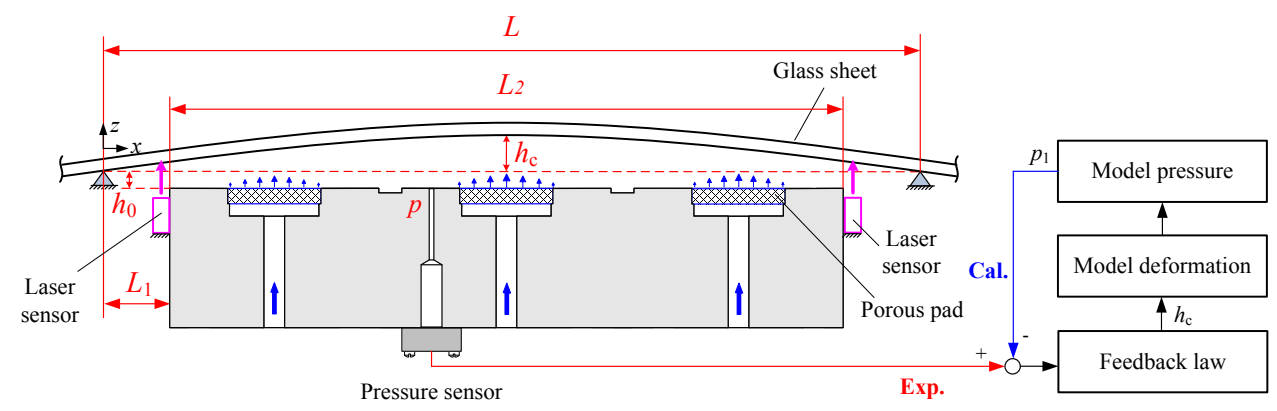

Figure 10 The principle of the one-dimensional estimating method
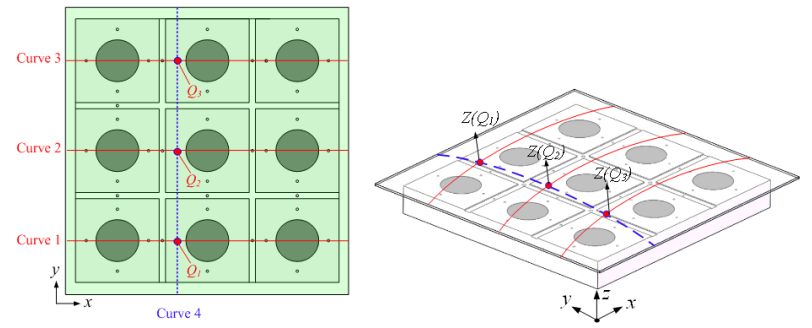

(a) Vertical view

(b) Axonometric drawing

Figure 11 The principle of the two-dimensional estimating method

An interpolation method is in use to obtain twodimensional deformation of the glass sheet. As shown in figure 11, the deformation of the curve 1, curve 2 and curve 3 , which are the centerlines of the three rows of the units, can be obtained with the method presented above. To estimate the deformation along y direction, for example, curve 4, three points on the three curves marked as $\mathrm{Q}_{1}\left(\mathrm{x}_{1}, \mathrm{y}_{1}\right), \mathrm{Q}_{2}\left(\mathrm{x}_{2}, \mathrm{y}_{2}\right)$ and $\mathrm{Q}_{3}\left(\mathrm{x}_{3}, \mathrm{y}_{3}\right)$ are selected. Then, with the three points, a parabolic curve $P(\mathrm{y})$ can be fit using equation (9). To be noticed that, with the three centerlines along $\mathrm{x}$-direction $\mathrm{P}(\mathrm{y})$ can be calculated, and inversely, with the centerlines along $\mathrm{y}$-direction $\mathrm{P}(\mathrm{x})$ can be calculated. Therefore, first, the deformation along $y$ direction is determined at an interval of $3 \mathrm{~mm}$, and second, the deformation along $\mathrm{x}$ direction is determined with the same interval. Then, at the overlapping points, the deformation values are averaged to improve the calculating accuracy.
$P(y)=\frac{\left(y-y_{2}\right)\left(y-y_{3}\right)}{\left(y_{1}-y_{2}\right)\left(y_{1}-y_{3}\right)} z\left(Q_{1}\right)+\frac{\left(y-y_{1}\right)\left(y-y_{3}\right)}{\left(y_{2}-y_{1}\right)\left(y_{1}-y_{3}\right)} z\left(Q_{2}\right)+\frac{\left(y-y_{1}\right)\left(y-y_{2}\right)}{\left(y_{3}-y_{1}\right)\left(y_{3}-y_{1}\right)} z\left(Q_{3}\right)$

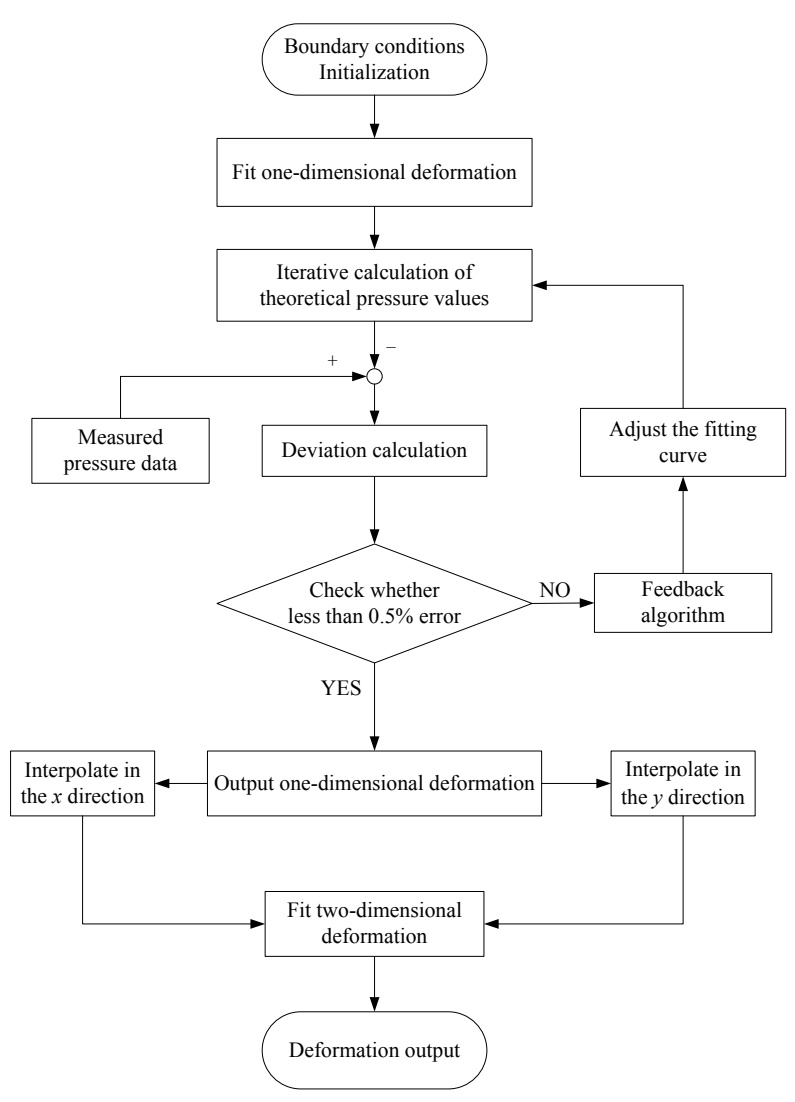

Figure 12 A flowchart indicating the estimation process 
Figure 12 shows the estimation procedure. Film pressure in the flange region is measured by pressure sensors which are connected to pressure taps. The measured pressure data is imported into the model and compared with the simulated results to shape the deformation curve by changing $h_{c}$. Frist, the simulations are initialized with actual conditions. Then, the film pressure is calculated using the iteration routine until it is sufficiently close to the measured data (within $0.5 \%$ error). During the process, During the process, a solution by means of proportional-integral algorithm (Eq. 10) is used to shape the estimated deformation. By this means, we can estimate the deformation of the centerline either along $\mathrm{x}$-direction or along $y$-direction. Interpolation operations are then applied with the deformation results on the centerlines to obtain the entire two-dimensional deformation.

$$
\Delta h_{c}=-K_{p}\left(e+K_{i} \sum e\right)
$$

where $\mathrm{K}_{\mathrm{p}}$ denotes the proportional gain, $\mathrm{K}_{\mathrm{i}}$ denotes the integral gain, e denotes the error, and $\Delta \mathrm{h}_{c}$ denotes the variation of the maximum deflection.
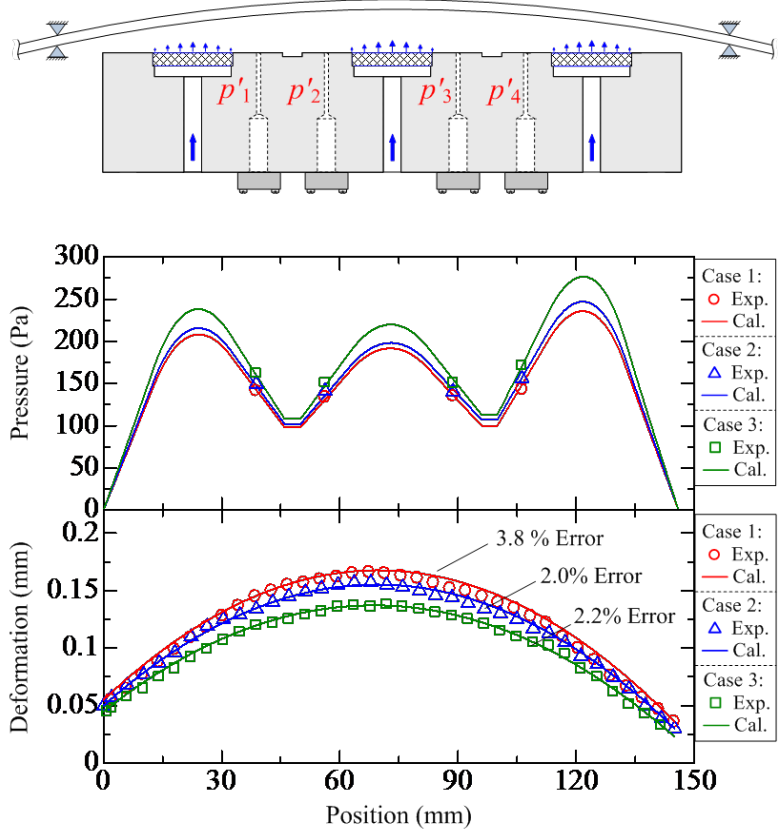

Figure 13 Estimated results of the deformation in comparison with the measured data at the centerline

Adjustment of the deformation shape can be easily implemented to make the calculated pressure close to the measured pressure with only one point used. However, if the pressure of the selected point is not accurately measured, it may cause a great error. Considering this, two points in the grooves are used as the boundary, and four inner pressure measuring points are used as the feedback to assure the reliability for the estimation. The deformation curve is shaped using a nonlinear least square method to take into consideration the error from every point. The estimation processes are conducted for the generated different degrees of deformation (three cases in total). In figure 13, the upper plot shows the calculated results in comparison with the measured film pressure, and the lower plot shows the corresponding deformation. The compressed air is supplied at a flow rate of $3 \mathrm{~L} / \mathrm{min}$ per unit. The deformation estimations are with an average error of $3.8 \%, 2.0 \%$ and $2.2 \%$ for case 1 , case 2 and case 3 , respectively.

Figure 14 shows the calculated results of the deformation at the six centerlines (three in $\mathrm{x}$-direction and three in y-direction), with which the two-dimensional deformation can be obtained. Figure 15 shows the entire status of the two-dimensional deformation in the case that the glass sheet is fixed at four corners, and observation shows that the center portion exhibits an obvious deformation.

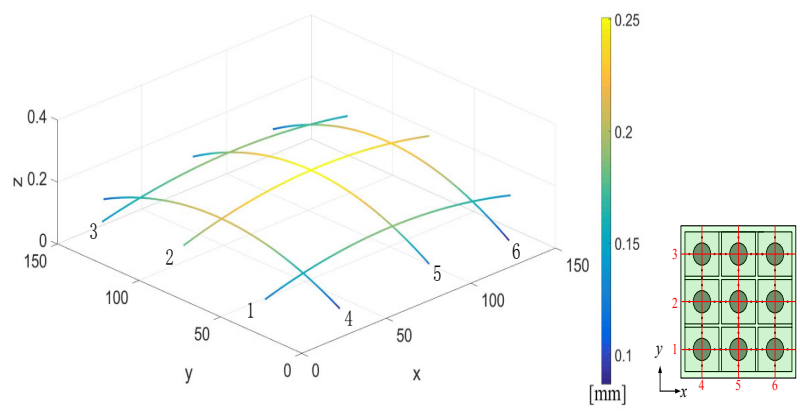

Figure 14 Estimated results of the deformation at all of the centerlines

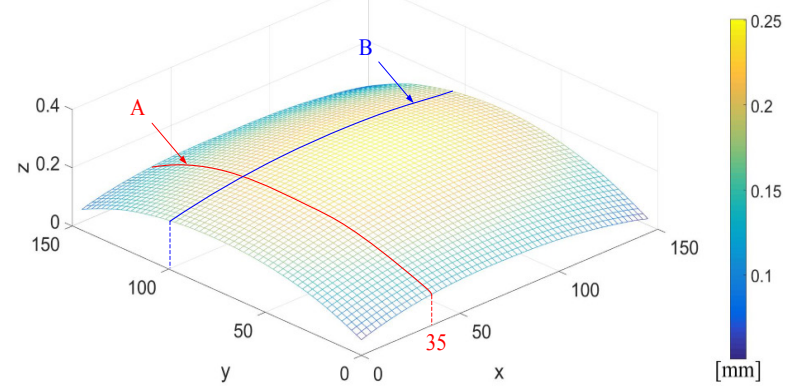

Figure 15 Estimated results of two-dimensional deformation

To verify the effectiveness of the estimating method, the results on the line that deviates from the centerline are also checked. That is, as shown in figure 15, the deformations of the glass sheet at the position $\mathrm{A}(\mathrm{x}=35$ $\mathrm{mm})$ and the position $B(y=100 \mathrm{~mm})$. Figure 16 compares the experimental results with the estimated results of the deformation curve A and B. The estimated results accord with the experimental data, with an average error around $3 \%$, indicating that the estimating method is feasible. The interpolation method can predict the deformations at the positions deviated from the centerline but one-dimensional estimation is not easy to realize. 


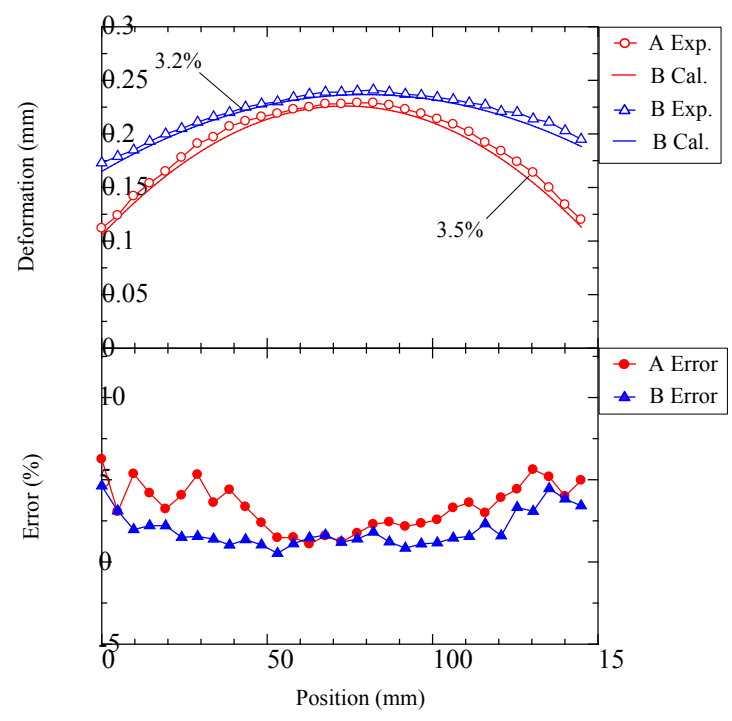

Figure 16 Calculated and experimental results of the deformation of the glass sheet and the errors

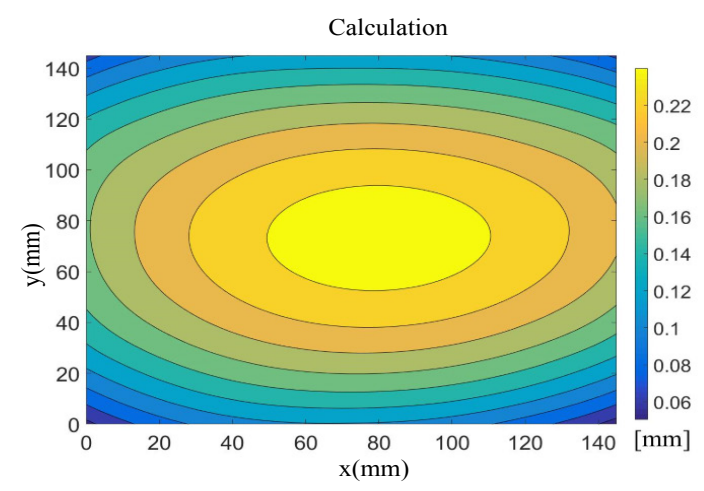

(a) Calculated results
Experiments are performed to measure the entire glass deformation with the apparatus shown in figure 6 . The glass sheet that has a size of $200 \mathrm{~mm} \times 155 \mathrm{~mm} \times 0.7$ $\mathrm{mm}$ is in use. Four supporting points distributed at four corners are employed, and the glass sheet is initially $150 \mu \mathrm{m}$ above the conveyor surface. The deformation with no air supply is treated as the reference, and the real result can be calculated by subtracted from the reference value. So, there is no deformation at the four supporting points and the center portion exhibits an apparent deformation. Figure 17 (a) and (b) show the contour plot of the calculated results and the measured results, respectively. Figure 18 shows the errors, and observation indicates that the errors are all within $4 \%$. Careful observation also shows that large errors appear at the position where the deformation exhibits a steep change. This is because the model cannot describe the flow behavior accurately. However, for the center area where there exists a large deformation, the estimating method works well since the glass sheet is relatively flat, without steep variations.

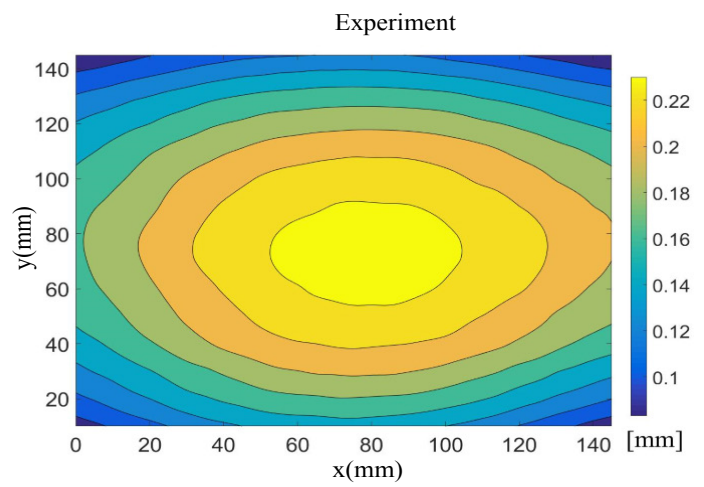

(b) Experimental results

Figure 17 Calculated and experimental results of the deformation of the glass sheet

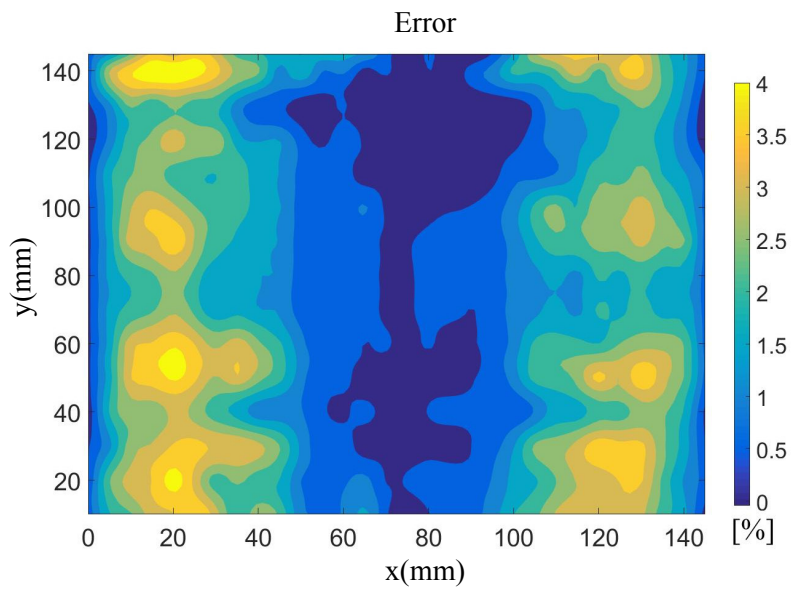

Figure 18 Errors between the Calculated and experimental results of the deformation of the glass sheet

\section{Conclusions}

A method for estimating the deformation of a glass sheet on a noncontact air conveyor by integration of measurement and simulation of the film pressure is proposed. A theoretical model is established within typical units, and the model is solved to attain the film pressure. An algorithm is used to adjust the deformed shape of the glass sheet with the measured film pressure to achieve that the calculated 
film pressure accords with the experimental data. Film pressure are monitored at several positions, and, the film pressure at the grooves are used as the boundary and that at the flange area are used as the feedback. Experimental setup is constructed to measure the two-dimensional deformation and the film pressure simultaneously. First, film pressure distributions are measured in a deformed and a non-deformed status, and it is observed that the calculated pressure shows good agreement with the experimental data. Then, the film pressure feedback method is used to estimate one-dimensional deformation on the centerlines along $\mathrm{x}$-direction and $\mathrm{y}$-direction, with which an interpolation operation is applied to obtain the two-dimensional deformation of the glass sheet. Experiments are performed to measure the deformation of the entire area. Comparison of the estimation results with the measured data indicates that the estimating method can afford a $4 \%$ accuracy. Furthermore, the estimating method shows a good performance for the area where the deformation exhibits a smooth variation. But for the area with a steep variation, the estimating results show relatively large errors.

Author Contributions: Conceptualization, Wei ZHONG; Data curation, Rui YANG; Investigation, Jiwen Fang; Methodology, Rui YANG and Chong LI; Resources, Chong LI; Validation, Jiwen Fang; Writing - original draft, Rui YANG; Writing - review \& editing, Wei ZHONG.

Conflict of Interest: The author(s) declare(s) that there is no conflict of interest regarding the publication of this paper.

Acknowledgments: This study was supported by the Natural Science Foundation of Jiangsu Province (Grant No. BK20181467) and the National Natural Science Foundation of China (Grant No. 51675247).

\section{References}

[1] Chen X.; Zhong W.; Li C.; Fang J.; Liu F. Development of a contactless air conveyor system for transporting and positioning planar objects. Micromachines 2018, 9(10), 487.

[2] Zhong W.; Ji X.; Li C.; Fang J.; Liu F. Determination of permeability and inertial coefficients of sintered metal porous media using an isothermal chamber. Appl. Sci 2018, 8(9), 1670.

[3] Shi K.; Li X.; Experimental and theoretical study of dynamic characteristics of Bernoulli gripper. Precis. Eng 2018, 52, 323-331.

[4] Savkiv V.; Mykhailyshyn R.; Duchon F. Gas dynamic analysis of the Bernoulli grippers interaction with the surface of flat objects with displacement of the center of mass. Vacuum 2019, 159, 524-34.

[5] Devitt D. The physics of glass flotation. Semicond. Int. Japan 2009, 5, 19-24.

[6] Im I.T.; Park C.W.; Kim K.S. A numerical study on the flow and heat transfer characteristics in a noncontact glass transportation unit. J. Mech. Sci. Technol. 2009, 23(12),

\section{6-3423.}

[7] Amano K.; Yoshimoto S.; Miyatake M.; Hirayama T. Basic investigation of noncontact transportation system for large TFT-LCD glass sheet used in CCD inspection section. Precis. Eng 2011, 35(1), 58-64.

[8] Oiwa N.; Masuda M.; Hirayama T.; Matsuoka T.; Yabe H. Deformation and flying height orbit of glass sheets on aerostatic porous bearing guides. Tribol. Int. 2012, 48, 2-7.

[9] Miyatake M.; Akahori H.; Yoshimoto S. Deformation of large liquid crystal display glass sheets across a gap between noncontact transportation devices. Precis. Eng. 2016, 46, 360-368.

[10] Funaki T.; Kawashima K.; Inoue S. Application of measurement integrated simulation to unsteady flow monitoring. IEEE 2006 SICE-ICASE International Joint Conference, Busan, Korea, 2006, pp. 5218-5221.

[11] Kontz M.E.; Book W.J.; Frankel J.G.; Pressure based exogenous force estimation. ASME 2006 Int. Mechanical Engineering Congress and Exposition, Chicago, Illinois, USA, 2006, pp.111-120.

[12] Yoon J.Y.; Singh R. Estimation of interfacial forces in a multi-degree of freedom isolation system using a dynamic load sensing mount and quasi-linear models. J. Sound Vib. 2011, 330(18-19), 4429-4446.

[13] Watanabe S.; Inoue H.; Fumoto K. An estimation of static aerodynamic forces of box girders using computational fluid dynamics. Wind Struct. 2004, 7(1), 29-40.

[14] Hayase T. F051001 Integration of Measurement and Simulation in Flow Analysis. 2011.

[15] Imagawa K.; Hayase T. Numerical experiment of measurement-integrated simulation to reproduce turbulent flows with feedback loop to dynamically compensate the solution using real flow information. Comput. Fluids 2010, 39(9), 1439-1450.

[16] Li X.; Horie M.; Kagawa T. Pressure-distribution methods for estimating lifting force of a swirl gripper. IEEE/ASME Trans. Mechatronics 2013, 19(2), 707-718.

[17] Nakao M.; Kawashima K.; Kagawa T. Measurement integrated simulation of wall pressure measurements using a turbulent model for analyzing oscillating orifice flow in a circular pipe. Comput. Fluids 2011, 49(1), 188196.

[18] Zhong W.; Gu X.; Li X.; Kagawa T. Study on the basic characteristics of a noncontact air conveyor for large glass sheets. Adv. Mech. Eng. 2017, 9(4), 1-13.

[19] Zhong W.; Li X.; Liu F.H.; Tao G.; Lu B.; Kagawa T. Measurement and correlation of pressure drop characteristics for air flow through sintered metal porous media. Transp. Porous Med.2014, 101(1), pp.53-67.

[20] Possamai F.C.; Ferreira R.T.S.; Prata A.T. Pressure distribution in laminar radial flow through inclined disks. Int. J. Heat Fluid Flow 2001, 22(4), 440-449.

[21] Li X.; Kawashima K.; Kagawa T. Analysis of vortex levitation. Exp. Therm. Fluid Sci. 2008, 32(8), 1448-1454. 